\title{
Improved Techniques for Grid Mapping with Rao-Blackwellized Particle Filters
}

\author{
Giorgio Grisetti* $^{* \dagger}$ Cyrill Stachniss ${ }^{\ddagger *} \quad$ Wolfram Burgard* \\ * University of Freiburg, Dept. of Computer Science, Georges-Köhler-Allee 79, D-79110 Freiburg, Germany \\ † Dipartimento Informatica e Sistemistica, Universitá "La Sapienza", Via Salaria 113, I-00198 Rome, Italy \\ ${ }^{\ddagger}$ Eidgenössische Technische Hochschule Zurich (ETH), IRIS, ASL, 8092 Zurich, Switzerland
}

\begin{abstract}
Recently, Rao-Blackwellized particle filters have been introduced as an effective means to solve the simultaneous localization and mapping problem. This approach uses a particle filter in which each particle carries an individual map of the environment. Accordingly, a key question is how to reduce the number of particles. In this paper, we present adaptive techniques for reducing this number in a Rao-Blackwellized particle filter for learning grid maps. We propose an approach to compute an accurate proposal distribution taking into account not only the movement of the robot but also the most recent observation. This drastically decreases the uncertainty about the robot's pose in the prediction step of the filter. Furthermore, we present an approach to selectively carry out resampling operations which seriously reduces the problem of particle depletion. Experimental results carried out with real mobile robots in large-scale indoor as well as in outdoor environments illustrate the advantages of our methods over previous approaches.
\end{abstract}

Index Terms-SLAM, Rao-Blackwellized particle filter, adaptive resampling, motion-model, improved proposal

\section{INTRODUCTION}

Building maps is one of the fundamental tasks of mobile robots. In the literature, the mobile robot mapping problem is often referred to as the simultaneous localization and mapping (SLAM) problem [4, 6, 9, 15, 16, 26, 29, 32, 39]. It is considered to be a complex problem, because for localization a robot needs a consistent map and for acquiring a map a robot requires a good estimate of its location. This mutual dependency between the pose and the map estimates makes the SLAM problem hard and requires searching for a solution in a high-dimensional space.

Murphy, Doucet, and colleagues [6, 32] introduced RaoBlackwellized particle filters as an effective means to solve the simultaneous localization and mapping problem. The main problem of the Rao-Blackwellized approaches is their complexity measured in terms of the number of particles required to build an accurate map. Therefore, reducing this quantity is one of the major challenges for this family of algorithms. Additionally, the resampling step can potentially eliminate the correct particle. This effect is also known as the particle depletion problem or as particle impoverishment [44].

In this work, we present two approaches to substantially increase the performance of Rao-Blackwellized particle filters applied to solve the SLAM problem with grid maps:

- A proposal distribution that considers the accuracy of the robot's sensors and allows us to draw particles in a highly accurate manner.
- An adaptive resampling technique which maintains a reasonable variety of particles and in this way enables the algorithm to learn an accurate map while reducing the risk of particle depletion.

The proposal distribution is computed by evaluating the likelihood around a particle-dependent most likely pose obtained by a scan-matching procedure combined with odometry information. In this way, the most recent sensor observation is taken into account for creating the next generation of particles. This allows us to estimate the state of the robot according to a more informed (and thus more accurate) model than the one obtained based only on the odometry information. The use of this refined model has two effects. The map is more accurate since the current observation is incorporated into the individual maps after considering its effect on the pose of the robot. This significantly reduces the estimation error so that less particles are required to represent the posterior. The second approach, the adaptive resampling strategy, allows us to perform a resampling step only when needed and in this way keeping a reasonable particle diversity. This results in a significantly reduced risk of particle depletion.

The work presented in this paper is an extension of our previous work [14] as it further optimizes the proposal distribution to even more accurately draw the next generation of particles. Furthermore, we added a complexity analysis, a formal description of the used techniques, and provide more detailed experiments in this paper. Our approach has been validated by a set of systematic experiments in large-scale indoor and outdoor environments. In all experiments, our approach generated highly accurate metric maps. Additionally, the number of the required particles is one order of magnitude lower than with previous approaches.

This paper is organized as follows. After explaining how a Rao-Blackwellized filter can be used in general to solve the SLAM problem, we describe our approach in Section III. We then provide implementation details in Section IV. Experiments carried out on real robots are presented in Section VI. Finally, Section VII discusses related approaches.

\section{Mapping With RAO-BlackWELlized PARTiCle FILTERS}

According to Murphy [32], the key idea of the RaoBlackwellized particle filter for SLAM is to estimate the joint posterior $p\left(x_{1: t}, m \mid z_{1: t}, u_{1: t-1}\right)$ about the map $m$ and the 
trajectory $x_{1: t}=x_{1}, \ldots, x_{t}$ of the robot. This estimation is performed given the observations $z_{1: t}=z_{1}, \ldots, z_{t}$ and the odometry measurements $u_{1: t-1}=u_{1}, \ldots, u_{t-1}$ obtained by the mobile robot. The Rao-Blackwellized particle filter for SLAM makes use of the following factorization

$$
\begin{aligned}
& p\left(x_{1: t}, m \mid z_{1: t}, u_{1: t-1}\right)= \\
& \quad p\left(m \mid x_{1: t}, z_{1: t}\right) \cdot p\left(x_{1: t} \mid z_{1: t}, u_{1: t-1}\right) .
\end{aligned}
$$

This factorization allows us to first estimate only the trajectory of the robot and then to compute the map given that trajectory. Since the map strongly depends on the pose estimate of the robot, this approach offers an efficient computation. This technique is often referred to as Rao-Blackwellization.

Typically, Eq. (1) can be calculated efficiently since the posterior over maps $p\left(m \mid x_{1: t}, z_{1: t}\right)$ can be computed analytically using "mapping with known poses" [31] since $x_{1: t}$ and $z_{1: t}$ are known.

To estimate the posterior $p\left(x_{1: t} \mid z_{1: t}, u_{1: t-1}\right)$ over the potential trajectories, one can apply a particle filter. Each particle represents a potential trajectory of the robot. Furthermore, an individual map is associated with each sample. The maps are built from the observations and the trajectory represented by the corresponding particle.

One of the most common particle filtering algorithms is the sampling importance resampling (SIR) filter. A RaoBlackwellized SIR filter for mapping incrementally processes the sensor observations and the odometry readings as they are available. It updates the set of samples that represents the posterior about the map and the trajectory of the vehicle. The process can be summarized by the following four steps:

1) Sampling: The next generation of particles $\left\{x_{t}^{(i)}\right\}$ is obtained from the generation $\left\{x_{t-1}^{(i)}\right\}$ by sampling from the proposal distribution $\pi$. Often, a probabilistic odometry motion model is used as the proposal distribution.

2) Importance Weighting: An individual importance weight $w_{t}^{(i)}$ is assigned to each particle according to the importance sampling principle

$$
w_{t}^{(i)}=\frac{p\left(x_{1: t}^{(i)} \mid z_{1: t}, u_{1: t-1}\right)}{\pi\left(x_{1: t}^{(i)} \mid z_{1: t}, u_{1: t-1}\right)} .
$$

The weights account for the fact that the proposal distribution $\pi$ is in general not equal to the target distribution of successor states.

3) Resampling: Particles are drawn with replacement proportional to their importance weight. This step is necessary since only a finite number of particles is used to approximate a continuous distribution. Furthermore, resampling allows us to apply a particle filter in situations in which the target distribution differs from the proposal. After resampling, all the particles have the same weight.

4) Map Estimation: For each particle, the corresponding map estimate $p\left(m^{(i)} \mid x_{1: t}^{(i)}, z_{1: t}\right)$ is computed based on the trajectory $x_{1: t}^{(i)}$ of that sample and the history of observations $z_{1: t}$.

The implementation of this schema requires to evaluate the weights of the trajectories from scratch whenever a new observation is available. Since the length of the trajectory increases over time, this procedure would lead to an obviously inefficient algorithm. According to Doucet et al. [7], we obtain a recursive formulation to compute the importance weights by restricting the proposal $\pi$ to fulfill the following assumption

$$
\begin{aligned}
\pi\left(x_{1: t} \mid z_{1: t}, u_{1: t-1}\right)= & \pi\left(x_{t} \mid x_{1: t-1}, z_{1: t}, u_{1: t-1}\right) \\
& \cdot \pi\left(x_{1: t-1} \mid z_{1: t-1}, u_{1: t-2}\right) .
\end{aligned}
$$

Based on Eq. (2) and (3), the weights are computed as

$$
\begin{aligned}
w_{t}^{(i)}= & \frac{p\left(x_{1: t}^{(i)} \mid z_{1: t}, u_{1: t-1}\right)}{\pi\left(x_{1: t}^{(i)} \mid z_{1: t}, u_{1: t-1}\right)} \\
= & \frac{\eta p\left(z_{t} \mid x_{1: t}^{(i)}, z_{1: t-1}\right) p\left(x_{t}^{(i)} \mid x_{t-1}^{(i)}, u_{t-1}\right)}{\pi\left(x_{t}^{(i)} \mid x_{1: t-1}^{(i)}, z_{1: t}, u_{1: t-1}\right)} \\
& \quad \underbrace{\frac{p\left(x_{1: t-1}^{(i)} \mid z_{1: t-1}, u_{1: t-2}\right)}{\pi\left(x_{1: t-1}^{(i)} \mid z_{1: t-1}, u_{1: t-2}\right)}}_{w_{t-1}^{(i)}} \\
\propto & \frac{p\left(z_{t} \mid m_{t-1}^{(i)}, x_{t}^{(i)}\right) p\left(x_{t}^{(i)} \mid x_{t-1}^{(i)}, u_{t-1}\right)}{\pi\left(x_{t} \mid x_{1: t-1}^{(i)}, z_{1: t}, u_{1: t-1}\right)} \cdot w_{t-1}^{(i)} .(6)
\end{aligned}
$$

Here $\eta=1 / p\left(z_{t} \mid z_{1: t-1}, u_{1: t-1}\right)$ is a normalization factor resulting from Bayes' rule that is equal for all particles.

Most of the existing particle filter applications rely on the recursive structure of Eq. (6). Whereas the generic algorithm specifies a framework that can be used for learning maps, it leaves open how the proposal distribution should be computed and when the resampling step should be carried out. Throughout the remainder of this paper, we describe a technique that computes an accurate proposal distribution and that adaptively performs resampling.

\section{RBPF WITH IMPROVED PROPOSALS AND ADAPTIVE RESAMPLING}

In the literature, several methods for computing improved proposal distributions and for reducing the risk of particle depletion have been proposed [7, 30, 35]. Our approach applies two concepts that have previously been identified as key pre-requisites for efficient particle filter implementations (see Doucet et al. [7]), namely the computation of an improved proposal distribution and an adaptive resampling technique.

\section{A. On the Improved Proposal Distribution}

As described in Section II, one needs to draw samples from a proposal distribution $\pi$ in the prediction step in order to obtain the next generation of particles. Intuitively, the better the proposal distribution approximates the target distribution, the better is the performance of the filter. For instance, if we were able to directly draw samples from the target distribution, the importance weights would become equal for all particles and the resampling step would no longer be needed. Unfortunately, in the context of SLAM a closed form of this posterior is not available in general.

As a result, typical particle filter applications [3, 29] use the odometry motion model as the proposal distribution. This motion model has the advantage that it is easy to compute for 


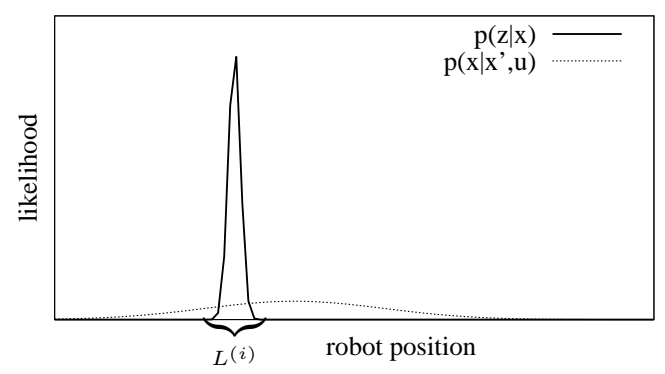

Fig. 1. The two components of the motion model. Within the interval $L^{(i)}$ the product of both functions is dominated by the observation likelihood in case an accurate sensor is used.

most types of robots. Furthermore, the importance weights are then computed according to the observation model $p\left(z_{t} \mid\right.$ $\left.m, x_{t}\right)$. This becomes clear by replacing $\pi$ in Eq. (6) by the motion model $p\left(x_{t} \mid x_{t-1}, u_{t-1}\right)$

$$
\begin{aligned}
w_{t}^{(i)} & =w_{t-1}^{(i)} \frac{\eta p\left(z_{t} \mid m_{t-1}^{(i)}, x_{t}^{(i)}\right) p\left(x_{t}^{(i)} \mid x_{t-1}^{(i)}, u_{t-1}\right)}{p\left(x_{t}^{(i)} \mid x_{t-1}^{(i)}, u_{t-1}\right)} \\
& \propto w_{t-1}^{(i)} \cdot p\left(z_{t} \mid m_{t-1}^{(i)}, x_{t}^{(i)}\right) .
\end{aligned}
$$

This proposal distribution, however, is suboptimal especially when the sensor information is significantly more precise than the motion estimate of the robot based on the odometry, which is typically the case if a robot equipped with a laser range finder (e.g., with a SICK LMS). Figure 1 illustrates a situation in which the meaningful area of the observation likelihood is substantially smaller than the meaningful area of the motion model. When using the odometry model as the proposal distribution in such a case, the importance weights of the individual samples can differ significantly from each other since only a fraction of the drawn samples cover the regions of state space that have a high likelihood under the observation model (area $L^{(i)}$ in Figure 1). As a result, one needs a comparably high number of samples to sufficiently cover the regions with high observation likelihood.

A common approach - especially in localization - is to use a smoothed likelihood function, which avoids that particles close to the meaningful area get a too low importance weight. However, this approach discards useful information gathered by the sensor and, at least to our experience, often leads to less accurate maps in the SLAM context.

To overcome this problem, one can consider the most recent sensor observation $z_{t}$ when generating the next generation of samples. By integrating $z_{t}$ into the proposal one can focus the sampling on the meaningful regions of the observation likelihood. According to Doucet [5], the distribution

$$
\begin{aligned}
& p\left(x_{t} \mid m_{t-1}^{(i)}, x_{t-1}^{(i)}, z_{t}, u_{t-1}\right)= \\
& \frac{p\left(z_{t} \mid m_{t-1}^{(i)}, x_{t}\right) p\left(x_{t} \mid x_{t-1}^{(i)}, u_{t-1}\right)}{p\left(z_{t} \mid m_{t-1}^{(i)}, x_{t-1}^{(i)}, u_{t-1}\right)}
\end{aligned}
$$

is the optimal proposal distribution with respect to the variance of the particle weights. Using that proposal, the computation of the weights turns into

$$
\begin{aligned}
w_{t}^{(i)} & =w_{t-1}^{(i)} \frac{\eta p\left(z_{t} \mid m_{t-1}^{(i)}, x_{t}^{(i)}\right) p\left(x_{t}^{(i)} \mid x_{t-1}^{(i)}, u_{t-1}\right)}{p\left(x_{t} \mid m_{t-1}^{(i)}, x_{t-1}^{(i)}, z_{t}, u_{t-1}\right)}(10) \\
& \propto w_{t-1}^{(i)} \frac{p\left(z_{t} \mid m_{t-1}^{(i)}, x_{t}^{(i)}\right) p\left(x_{t}^{(i)} \mid x_{t-1}^{(i)}, u_{t-1}\right)}{\frac{p\left(z_{t} \mid m_{t-1}^{(i)}, x_{t}\right) p\left(x_{t} \mid x_{t-1}^{(i)}, u_{t-1}\right)}{p\left(z_{t} \mid m_{t-1}^{(i)}, x_{t-1}^{(i)}, u_{t-1}\right)}} \\
& =w_{t-1}^{(i)} \cdot p\left(z_{t} \mid m_{t-1}^{(i)}, x_{t-1}^{(i)}, u_{t-1}\right) \\
& =w_{t-1}^{(i)} \cdot \int p\left(z_{t} \mid x^{\prime}\right) p\left(x^{\prime} \mid x_{t-1}^{(i)}, u_{t-1}\right) d x^{\prime} .
\end{aligned}
$$

When modeling a mobile robot equipped with an accurate sensor like, e.g., a laser range finder, it is convenient to use such an improved proposal since the accuracy of the laser range finder leads to extremely peaked likelihood functions. In the context of landmark-based SLAM, Montemerlo et al. [26] presented a Rao-Blackwellized particle filter that uses a Gaussian approximation of the improved proposal. This Gaussian is computed for each particle using a Kalman filter that estimates the pose of the robot. This approach can be used when the map is represented by a set of features and if the error affecting the feature detection is assumed to be Gaussian. In this work, we transfer the idea of computing an improved proposal to the situation in which dense grid maps are used instead of landmark-based representations.

\section{B. Efficient Computation of the Improved Proposal}

When modeling the environment with grid maps, a closed form approximation of an informed proposal is not directly available due to the unpredictable shape of the observation likelihood function.

In theory, an approximated form of the informed proposal can be obtained using the adapted particle filter [35]. In this framework, the proposal for each particle is constructed by computing a sampled estimate of the optimal proposal given in Eq. (9). In the SLAM context, one would first have to sample a set of potential poses $x_{j}$ of the robot from the motion model $p\left(x_{t} \mid x_{t-1}^{(i)}, u_{t-1}\right)$. In a second step, these samples need to be weighed by the observation likelihood to obtain an approximation of the optimal proposal. However, if the observation likelihood is peaked the number of pose samples $x_{j}$ that has to be sampled from the motion model is high, since a dense sampling is needed for sufficiently capturing the typically small areas of high likelihood. This results in a similar problem than using the motion model as the proposal: a high number of samples is needed to sufficiently cover the meaningful region of the distribution.

One of our observations is that in the majority of cases the target distribution has only a limited number of maxima and it mostly has only a single one. This allows us to sample positions $x_{j}$ covering only the area surrounding these maxima. Ignoring the less meaningful regions of the distribution saves a significant amount of computational resources since it requires less samples. In the previous version of this work [14], we approximated $p\left(x_{t} \mid x_{t-1}^{(i)}, u_{t-1}\right)$ by a constant $k$ within the interval $L^{(i)}$ (see also Figure 1) given by

$$
L^{(i)}=\left\{x \mid p\left(z_{t} \mid m_{t-1}^{(i)}, x\right)>\epsilon\right\} .
$$




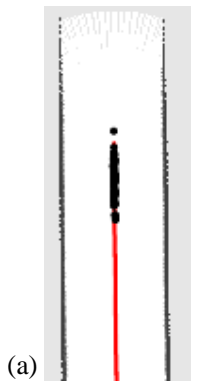

(b)
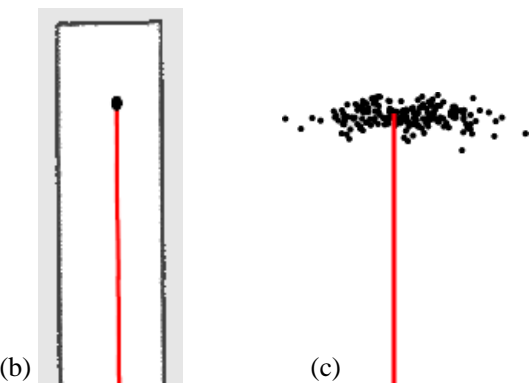

Fig. 2. Particle distributions typically observed during mapping. In an open corridor, the particles distribute along the corridor (a). In a dead end corridor the uncertainty is small in all dimensions (b). Such posteriors are obtained because we explicitely take into account the most recent observation when sampling the next generation of particles. In contrast to that, the raw odometry motion model leads less peaked posteriors (c).

In our current approach, we consider both components, the observation likelihood and the motion model within that interval $L^{(i)}$. We locally approximate the posterior $p\left(x_{t}\right.$ $\left.m_{t-1}^{(i)}, x_{t-1}^{(i)}, z_{t}, u_{t-1}\right)$ around the maximum of the likelihood function reported by a scan registration procedure.

To efficiently draw the next generation of samples, we compute a Gaussian approximation $\mathcal{N}$ based on that data. The main differences to previous approaches is that we first use a scan-matcher to determine the meaningful area of the observation likelihood function. We then sample in that meaningful area and evaluate the sampled points based on the target distribution. For each particle $i$, the parameters $\mu_{t}^{(i)}$ and $\Sigma_{t}^{(i)}$ are determined individually for $K$ sampled points $\left\{x_{j}\right\}$ in the interval $L^{(i)}$. We furthermore take into account the odometry information when computing the mean $\mu^{(i)}$ and the variance $\Sigma^{(i)}$. We estimate the Gaussian parameters as

$$
\begin{aligned}
\mu_{t}^{(i)}= & \frac{1}{\eta^{(i)}} \cdot \sum_{j=1}^{K} x_{j} \cdot p\left(z_{t} \mid m_{t-1}^{(i)}, x_{j}\right) \\
& \cdot p\left(x_{j} \mid x_{t-1}^{(i)}, u_{t-1}\right) \\
\Sigma_{t}^{(i)}= & \frac{1}{\eta^{(i)}} \cdot \sum_{j=1}^{K} p\left(z_{t} \mid m_{t-1}^{(i)}, x_{j}\right) \\
& \cdot p\left(x_{j} \mid x_{t-1}^{(i)}, u_{t-1}\right) \\
& \cdot\left(x_{j}-\mu_{t}^{(i)}\right)\left(x_{j}-\mu_{t}^{(i)}\right)^{T}
\end{aligned}
$$

with the normalization factor

$$
\eta^{(i)}=\sum_{j=1}^{K} p\left(z_{t} \mid m_{t-1}^{(i)}, x_{j}\right) \cdot p\left(x_{j} \mid x_{t-1}^{(i)}, u_{t-1}\right) .
$$

In this way, we obtain a closed form approximation of the optimal proposal which enables us to efficiently obtain the next generation of particles. Using this proposal distribution, the weights can be computed as

$$
\begin{aligned}
w_{t}^{(i)} & =w_{t-1}^{(i)} \cdot p\left(z_{t} \mid m_{t-1}^{(i)}, x_{t-1}^{(i)}, u_{t-1}\right) \\
& =w_{t-1}^{(i)} \cdot \int p\left(z_{t} \mid m_{t-1}^{(i)}, x^{\prime}\right) \cdot p\left(x^{\prime} \mid x_{t-1}^{(i)}, u_{t-1}\right) d x \\
& \simeq w_{t-1}^{(i)} \cdot \sum_{j=1}^{K} p\left(z_{t} \mid m_{t-1}^{(i)}, x_{j}\right) \cdot p\left(x_{j} \mid x_{t-1}^{(i)}, u_{t-1}\right)
\end{aligned}
$$

$$
\text { compros }
$$
robot which is illustrated in Figure 2 (c) is used as a proposal. Note that such situations occur rarely in real datasets (see also Section VI-E).

\section{Discussion about the Improved Proposal}

The computations presented in this section enable us to determine the parameters of a Gaussian proposal distribution for each particle individually. The proposal takes into account the most recent odometry reading and laser observation while at the same time allowing us efficient sampling. The resulting densities have a much lower uncertainty compared to situations in which the odometry motion model is used. To illustrate this fact, Figure 2 depicts typical particle distributions obtained with our approach. In case of a straight featureless corridor, the samples are typically spread along the main direction of the corridor as depicted in Figure 2 (a). Figure 2 (b) illustrates the robot reaching the end of such a corridor. As can be seen, he uncertainty in the direction of the corridor decreases and all samples are centered around a single point. In contrast to that, Figure 2 (c) shows the resulting distribution when sampling from the raw motion model.

As explained above, we use a scan-matcher to determine the mode of the meaningful area of the observation likelihood function. In this way, we focus the sampling on the important regions. Most existing scan-matching algorithms maximize the observation likelihood given a map and an initial guess of the robot's pose. When the likelihood function is multi-modal, which can occur when, e.g., closing a loop, the scan-matcher returns for each particle the maximum which is closest to the initial guess. In general, it can happen that additional maxima in the likelihood function are missed since only a single mode is reported. However, since we perform frequent filter updates (after each movement of $0.5 \mathrm{~m}$ or a rotation of $25^{\circ}$ ) and imit the search area of the scan-matcher, we consider that the distribution has only a single mode when sampling data points to compute the Gaussian proposal. Note that in situations like a loop closure, the filter is still able to keep multiple hypotheses because the initial guess for the starting position of the scanmatcher when reentering a loop is different for each particle.

Nevertheless, there are situations in which the filter can - at least in theory - become overly confident. This might happen in extremely cluttered environments and when the odometry is highly affected by noise. A solution to this problem is to track the multiple modes of the scan-matcher and repeat the sampling process separately for each node. However, in our experiments carried out using real robots we never encountered uch a situation.

During filtering, it can happen that the scan-matching process fails because of poor observations or a too small (1) 


\section{Adaptive Resampling}

A further aspect that has a major influence on the performance of a particle filter is the resampling step. During resampling, particles with a low importance weight $w^{(i)}$ are typically replaced by samples with a high weight. On the one hand, resampling is necessary since only a finite number of particles are used to approximate the target distribution. On the other hand, the resampling step can remove good samples from the filter which can lead to particle impoverishment. Accordingly, it is important to find a criterion for deciding when to perform the resampling step. Liu [23] introduced the so-called effective sample size to estimate how well the current particle set represents the target posterior. In this work, we compute this quantity according to the formulation of Doucet et al. [7] as

$$
N_{\text {eff }}=\frac{1}{\sum_{i=1}^{N}\left(\tilde{w}^{(i)}\right)^{2}},
$$

where $\tilde{w}^{(i)}$ refers to the normalized weight of particle $i$.

The intuition behind $N_{\text {eff }}$ is as follows. If the samples were drawn from the target distribution, their importance weights would be equal to each other due to the importance sampling principle. The worse the approximation of the target distribution, the higher is the variance of the importance weights. Since $N_{\text {eff }}$ can be regarded as a measure of the dispersion of the importance weights, it is a useful measure to evaluate how well the particle set approximates the target posterior. Our algorithm follows the approach proposed by Doucet et al. [7] to determine whether or not the resampling step should be carried out. We resample each time $N_{\text {eff }}$ drops below the threshold of $N / 2$ where $N$ is the number of particles. In extensive experiments, we found that this approach drastically reduces the risk of replacing good particles, because the number of resampling operations is reduced and they are only performed when needed.

\section{E. Algorithm}

The overall process is summarized in Algorithm 1. Each time a new measurement tuple $\left(u_{t-1}, z_{t}\right)$ is available, the proposal is computed for each particle individually and is then used to update that particle. This results in the following steps:

1) An initial guess $x_{t}^{(i)}=x_{t-1}^{(i)} \oplus u_{t-1}$ for the robot's pose represented by the particle $i$ is obtained from the previous pose $x_{t-1}^{(i)}$ of that particle and the odometry measurements $u_{t-1}$ collected since the last filter update. Here, the operator $\oplus$ corresponds to the standard pose compounding operator [24].

2) A scan-matching algorithm is executed based on the map $m_{t-1}^{(i)}$ starting from the initial guess $x_{t}^{\prime(i)}$. The search performed by the scan-matcher is bounded to a limited region around $x_{t}^{\prime(i)}$. If the scan-matching reports a failure, the pose and the weights are computed according to the motion model (and the steps 3 and 4 are ignored).

3) A set of sampling points is selected in an interval around the pose $\hat{x}_{t}^{(i)}$ reported scan-matcher. Based on this points, the mean and the covariance matrix of the proposal are computed by pointwise evaluating the target distribution $p\left(z_{t} \mid m_{t-1}^{(i)}, x_{j}\right) p\left(x_{j} \mid x_{t-1}^{(i)}, u_{t-1}\right)$ in the sampled positions $x_{j}$. During this phase, also the weighting factor $\eta^{(i)}$ is computed according to Eq. (17).

4) The new pose $x_{t}^{(i)}$ of the particle $i$ is drawn from the Gaussian approximation $\mathcal{N}\left(\mu_{t}^{(i)}, \Sigma_{t}^{(i)}\right)$ of the improved proposal distribution.

5) Update of the importance weights.

6) The map $m^{(i)}$ of particle $i$ is updated according to the drawn pose $x_{t}^{(i)}$ and the observation $z_{t}$.

After computing the next generation of samples, a resampling step is carried out depending on the value of $N_{\text {eff }}$.

\section{IMPLEMENTATION ISSUES}

This section provides additional information about implementation details used in our current system. These issues are not required for the understanding of the general approach but complete the precise description of our mapping system. In the following, we briefly explain the used scan-matching approach, the observation model, and how to pointwise evaluate the motion model.

Our approach applies a scan-matching technique on a per particle basis. In general, an arbitrary scan-matching technique can be used. In our implementation, we use the scan-matcher "vasco" which is part of the Carnegie Mellon Robot Navigation Toolkit (CARMEN) [27, 36]. This scan-matcher aims to find the most likely pose by matching the current observation against the map constructed so far

$$
\hat{x}_{t}^{(i)}=\underset{x}{\operatorname{argmax}} p\left(x \mid m_{t-1}^{(i)}, z_{t}, x_{t}^{(i)}\right),
$$

where $x_{t}^{\prime(i)}$ is the initial guess. The scan-matching technique performs a gradient descent search on the likelihood function of the current observation given the grid map. Note that in our mapping approach, the scan-matcher is only used for finding the local maximum of the observation likelihood function. In practice, any scan-matching technique which is able to compute the best alignment between a reference map $m_{t-1}^{(i)}$ and the current scan $z_{t}$ given an initial guess $x_{t}^{\prime(i)}$ can be used.

In order to solve Eq. (21), one applies Bayes' rule and seeks for the pose with the highest observation likelihood $p\left(z_{t} \mid m, x\right)$. To compute the likelihood of an observation, we use the so called "beam endpoint model" [40]. In this model, the individual beams within a scan are considered to be independent. Furthermore, the likelihood of a beam is computed based on the distance between the endpoint of the beam and the closest obstacle from that point. To achieve a fast computation, one typically uses a convolved local grid map.

Additionally, the construction of our proposal requires to evaluate $p\left(z_{t} \mid m_{t-1}^{(i)}, x_{j}\right) p\left(x_{j} \mid x_{t-1}^{(i)}, u_{t-1}\right)$ at the sampled points $x_{j}$. We compute the first component according to the previously mentioned "beam endpoint model". To evaluate the second term, several closed form solutions for the motion estimate are available. The different approaches mainly differ in the way the kinematics of the robot are modeled. In our current implementation, we compute $p\left(x_{j} \mid x_{t-1}, u_{t-1}\right)$ according to 


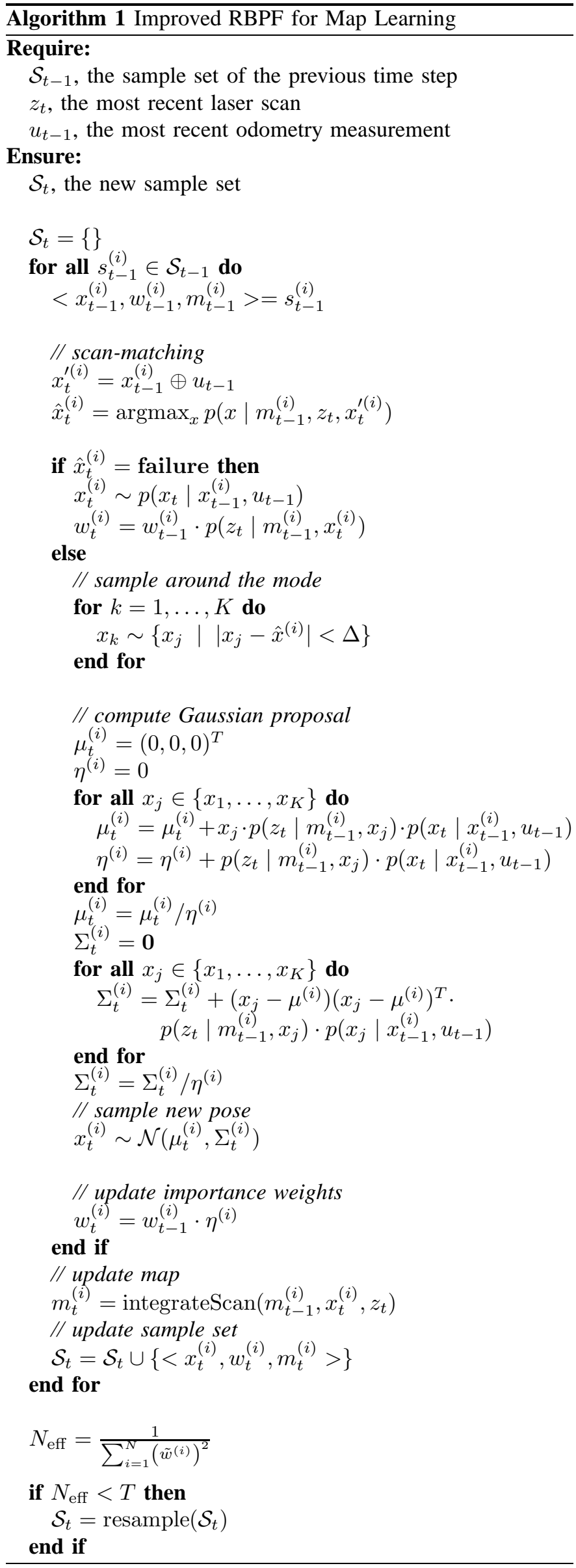

the Gaussian approximation of the odometry motion model described in [41]. We obtain this approximation through Taylor expansion in an EKF-style procedure. In general, there are more sophisticated techniques estimating the motion of the robot. However, we use that model to estimate a movement between two filter updates which is performed after the robot traveled around $0.5 \mathrm{~m}$. In this case, this approximation works well and we did not observed a significant difference between the EKF-like model and the in general more accurate samplebased velocity motion model [41].

\section{COMPlexity}

This section discusses the complexity of the presented approach to learn grid maps with a Rao-Blackwellized particle filter. Since our approach uses a sample set to represent the posterior about maps and poses, the number $N$ of samples is the central quantity. To compute the proposal distribution, our approach samples around the most likely position reported by the scan-matcher. This sampling is done for each particle a constant number of times $(K)$ and there is no dependency between the particles when computing the proposal. Furthermore, the most recent observation $z_{t}$ used to compute $\mu^{(i)}$ and $\Sigma^{(i)}$ covers only an area of the map $m$ (bounded by the odometry error and the maximum range of the sensor), so the complexity depends only on the number $N$ of particles. The same holds for the update of the individual maps associated to each of the particles.

During the resampling step, the information associated to a particle needs to be copied. In the worst case, $N-1$ samples are replaced by a single particle. In our current system, each particle stores and maintains its own grid map. To duplicate a particle, we therefore have to copy the whole map. As a result, a resampling action introduces a worst case complexity of $O(N M)$, where $M$ is the size of the corresponding grid map. However, using the adaptive resampling technique, only very few resampling steps are required during mapping.

To decide whether or not a resampling is needed, the effective sample size (see Eq. (20)) needs to be taken into account. Again, the computation of the quantity introduces a complexity of $O(N)$.

As a result, if no resampling operation is required, the overall complexity for integrating a single observation depends only linearly on the number of particles. If a resampling is required, the additional factor $M$ which represents the size of the map is introduced and leads to a complexity of $O(N M)$. The complexity of each individual operation is depicted in Table I.

Note that the complexity of the resampling step can be reduced by using a more intelligent map representation as done in DP-SLAM [9]. It can be shown, that in this case the complexity of a resampling step is reduced to $O\left(A N^{2} \log N\right)$, where $A$ is the area covered by the sensor. However, building an improved map representation is not the aim of this paper. We actually see our approach as orthogonal to DP-SLAM because both techniques can be combined. Furthermore, in our experiments using real world data sets, we figured out the resampling steps are not the dominant part and they occur rarely due to the adaptive resampling strategy. 
TABLE I

COMPLEXITY OF THE DIFFERENT OPERATIONS FOR INTEGRATING ONE OBSERVATION.

\begin{tabular}{|l|l|}
\hline Operation & Complexity \\
\hline Computation of the proposal distribution & $O(N)$ \\
\hline Update of the grid map & $O(N)$ \\
\hline Computation of the weights & $O(N)$ \\
\hline Test if resampling is required & $O(N)$ \\
\hline Resampling & $O(N M)$ \\
\hline
\end{tabular}
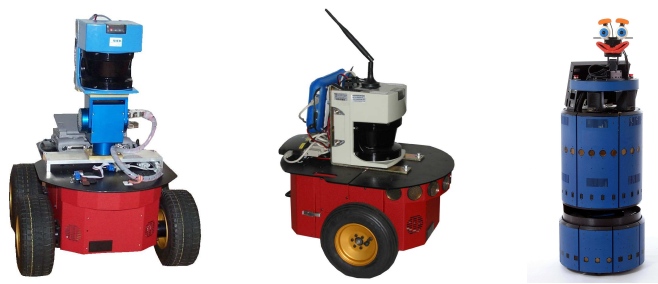

Fig. 3. Different types of robot used to acquire real robot data used for mapping (ActivMedia Pioneer 2 AT, Pioneer 2 DX-8, and an iRobot B21r).

\section{EXPERIMENTS}

The approach described above has been implemented and tested using real robots and datasets gathered with real robots. Our mapping approach runs online on several platforms like ActivMedia Pioneer2 AT, Pioneer 2 DX-8, and iRobot B21r robots equipped with a SICK LMS and PLS laser range finders (see Figure 3). The experiments have been carried out in a variety of environments and showed the effectiveness of our approach in indoor and outdoor settings. Most of the maps generated by our approach can be magnified up to a resolution of $1 \mathrm{~cm}$, without observing considerable inconsistencies. Even in big real world datasets covering an area of approximately $250 \mathrm{~m}$ by $250 \mathrm{~m}$, our approach never required more than 80 particles to build accurate maps. In the reminder of this section, we discuss the behavior of the filter in different datasets. Furthermore, we give a quantitative analysis of the performance of the presented approach. Highly accurate grid maps have been generated with our approach from several datasets. These maps, raw data files, and an efficient implementation of our mapping system are available on the web [38].

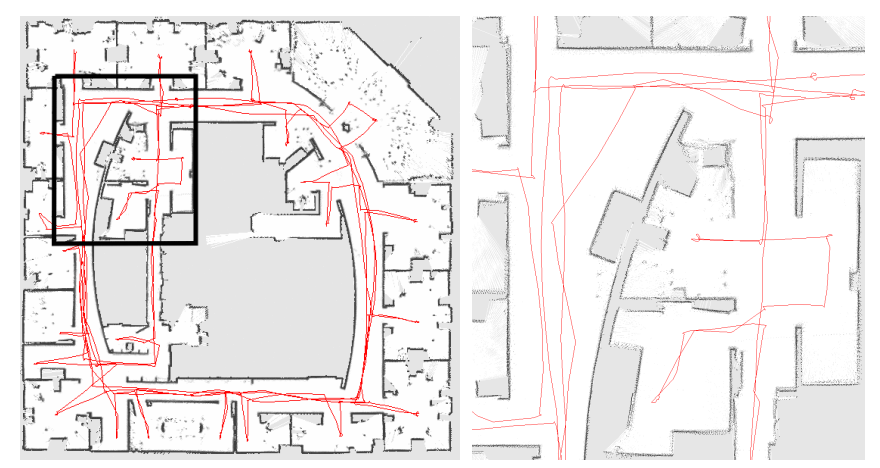

Fig. 4. The Intel Research Lab. The robot starts in the upper part of the circular corridor, and runs several times around the loop, before entering the rooms. The left image depicts the resulting map generated with 15 particles. The right image shows a cut-out with $1 \mathrm{~cm}$ grid resolution to illustrate the accuracy of the map in the loop closure point.

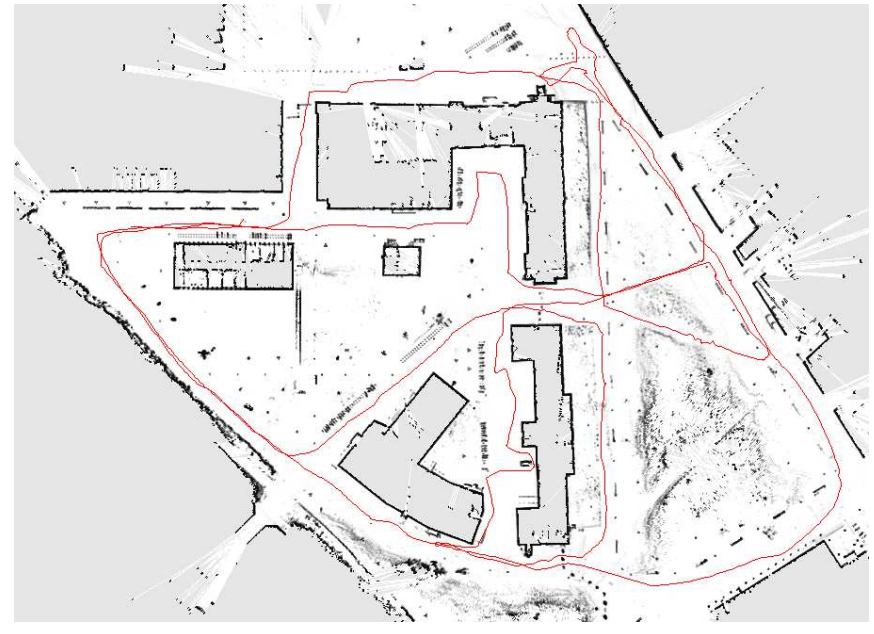

Fig. 5. The Freiburg Campus. The robot first runs around the external perimeter in order to close the outer loop. Afterwards, the internal parts of the campus are visited. The overall trajectory has a length of $1.75 \mathrm{~km}$ and covers an area of approximately $250 \mathrm{~m}$ by $250 \mathrm{~m}$. The depicted map was generated using 30 particles.

\section{A. Mapping Results}

The datasets discussed here have been recorded at the Intel Research Lab in Seattle, at the campus of the University of Freiburg, and at the Killian Court at MIT. The maps of these environments are depicted in Figures 4, 5, and 6.

a) Intel Research Lab: The Intel Research Lab is depicted in the left image of Figure 4 and has a size of $28 \mathrm{~m}$ by $28 \mathrm{~m}$. The dataset has been recorded with a Pioneer II robot equipped with a SICK sensor. To successfully correct this dataset, our algorithm needed only 15 particles. As can be seen in the right image of Figure 4, the quality of the final map is so high that the map can be magnified up to $1 \mathrm{~cm}$ of resolution without showing any significant errors.

b) Freiburg Campus: The second dataset has been recorded outdoors at the Freiburg campus. Our system needed only 30 particles to produce a good quality map such as the one shown in Figure 5. Note that this environment partly violates the assumptions that the environment is planar. Additionally, there were objects like bushes and grass as well as moving objects like cars and people. Despite the resulting spurious measurements, our algorithm was able to generate an accurate map.

c) MIT Killian Court: The third experiment was performed with a dataset acquired at the MIT Killian court ${ }^{1}$ and the resulting map is depicted in Figure 6. This dataset is extremely challenging since it contains several nested loops, which can cause a Rao-Blackwellized particle filter to fail due to particle depletion. Using this dataset, the selective resampling procedure turned out to be important. A consistent and topologically correct map can be generated with 60 particles. However, the resulting maps sometimes show artificial double walls. By employing 80 particles it is possible to achieve high quality maps.

\footnotetext{
${ }^{1}$ Note that there exist two different versions of that dataset on the web. One has a pre-corrected odometry and the other one has not. We used the raw version without pre-corrected odometry information.
} 


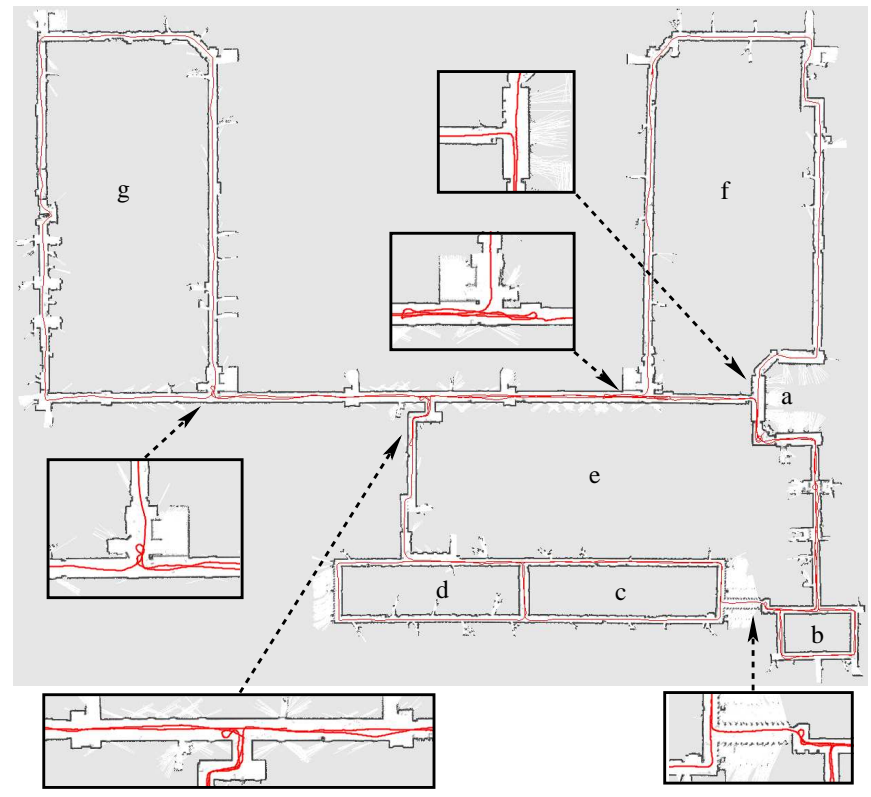

Fig. 6. The MIT Killian Court. The robot starts from the point labeled $a$ and then traverses the first loop labeled $b$. It then moves through the loops labeled $c, d$ and moves back to the place labeled $a$ and the loop labeled $b$. It the visits the two big loops labeled $f$ and $g$. The environment has a size of $250 \mathrm{~m}$ by $215 \mathrm{~m}$ and the robot traveled $1.9 \mathrm{~km}$. The depicted map has been generated with 80 particles. The rectangles show magnifications of several parts of the map.

TABLE II

THE NUMBER OF PARTICLES NEEDED BY OUR ALGORITHM COMPARED TO THE APPROACH OF HÄHNEL et al. [16].

\begin{tabular}{|c|c|c|c|}
\hline Proposal Distribution & Intel & MIT & Freiburg \\
\hline our approach & 8 & 60 & 20 \\
\hline approach of [16] & 40 & 400 & 400 \\
\hline
\end{tabular}

\section{B. Quantitative Results}

In order to measure the improvement in terms of the number of particles, we compared the performance of our system using the informed proposal distribution to the approach done by Hähnel et al. [16]. Table II summarizes the number of particles needed by a RBPF for providing a topologically correct map in at least $60 \%$ of all applications of our algorithm.

It turns out that in all of the cases, the number of particles required by our approach was approximately one order of magnitude smaller than the one required by the other approach. Moreover, the resulting maps are better due to our improved sampling process that takes the last reading into account.

Figure 7 summarizes results about the success ratio of our

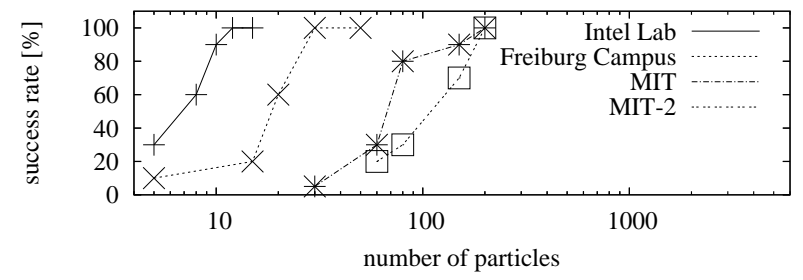

Fig. 7. Success rate of our algorithm in different environments depending on the number of particles. Each success rate was determined using 20 runs. For the experiment MIT- 2 we disabled the adaptive resampling.

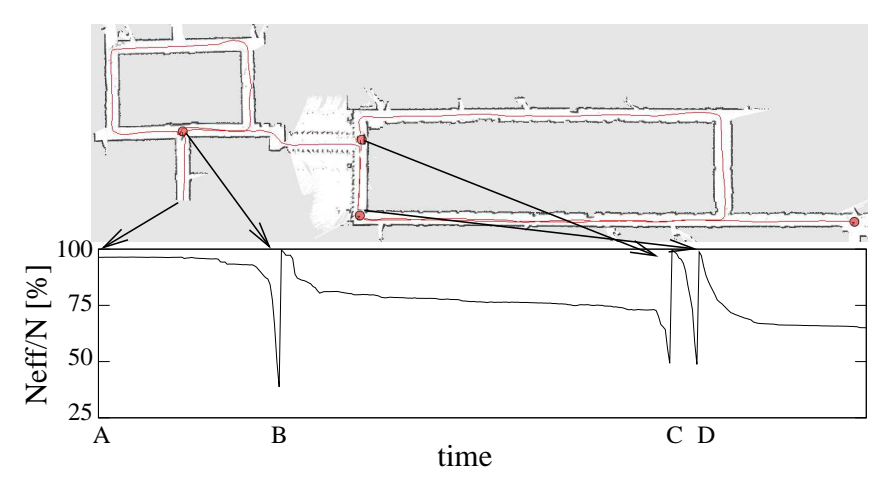

Fig. 8. The graph plots the evolution of the $N_{\text {eff }}$ function over time during an experiment in the environment shown in the top image. At time B the robot closes the small loop. At time $\mathrm{C}$ and $\mathrm{D}$ resampling actions are carried after the robots closes the big loop.

algorithm in the environments considered here. The plots show the percentage of correctly generated maps, depending on the number of particles used. The question if a map is consistent or not has been evaluated by visual inspection in a blind fashion (the inspectors were not the authors). As a measure of success, we used the topological correctness.

\section{Effects of Improved Proposals and Adaptive Resampling}

The increased performance of our approach is due to the interplay of two factors, namely the improved proposal distribution, which allows us to generate samples with an high likelihood, and the adaptive resampling controlled by monitoring $N_{\text {eff }}$. For proposals that do not consider the whole input history, it has been proven that $N_{\text {eff }}$ can only decrease (stochastically) over time [7]. Only after a resampling operation, $N_{\text {eff }}$ recovers its maximum value. It is important to notice that the behavior of $N_{\text {eff }}$ depends on the proposal: the worse the proposal, the faster $N_{\text {eff }}$ drops.

We found that the evolution of $N_{\text {eff }}$ using our proposal distribution shows three different behaviors depending on the information obtained from the robot's sensors. Figure 8 illustrates the evolution of $N_{\text {eff }}$ during an experiment. Whenever the robot moves through unknown terrain, $N_{\text {eff }}$ typically drops slowly. This is because the proposal distribution becomes less peaked and the likelihoods of observations often differ slightly. The second behavior can be observed when the robot moves through a known area. In this case, each particle keeps localized within its own map due to the improved proposal distribution and the weights are more or less equal. This results in a more or less constant behavior of $N_{\text {eff }}$. Finally, when closing a loop, some particles are correctly aligned with their map while others are not. The correct particles have a high weight, while the wrong ones have a low weight. Thus the variance of the importance weights increases and $N_{\text {eff }}$ substantially drops.

Accordingly, the threshold criterion applied on $N_{\text {eff }}$ typically forces a resampling action when the robot is closing a loop. In all other cases, the resampling is avoided and in this way the filter keeps a variety of samples in the particle set. As a result, the risk of particle depletion problem is seriously reduced. To analyze this, we performed an experiment in 

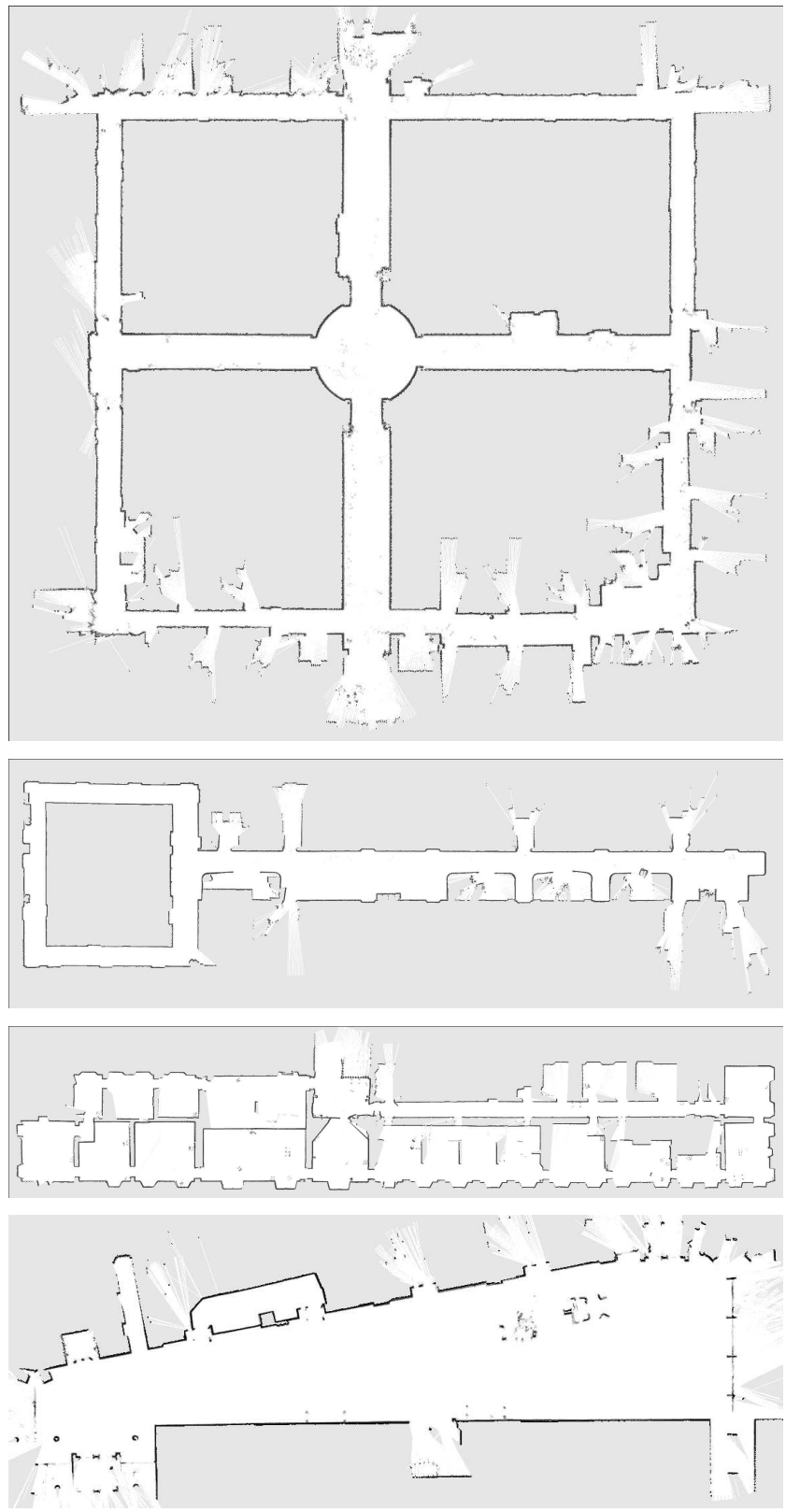

Fig. 9. Maps from the ACES building at University of Texas, the 4th floor of the Sieg Hall at the University of Washington, the Belgioioso building, and building 101 at the University of Freiburg.

which we compared the success rate of our algorithm to that of a particle filter which resamples at every step. As Figure 7 illustrates, our approach more often converged to the correct solution (MIT curve) for the MIT dataset compared to the particle filter with the same number of particles and a fixed resampling strategy (MIT-2 curve).

To give a more detailed impression about the accuracy of our new mapping technique, Figure 9 depicts maps learned from well known and freely available [18] real robot datasets recorded at the University of Texas, at the University of Washington, at Belgioioso, and at the University of Freiburg. Each map was built using 30 particles to represent the posterior about maps and poses.
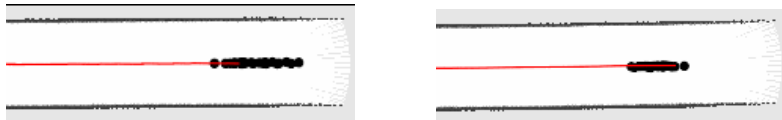

Fig. 10. The effect of considering the odometry in the computation of the proposal on the particle cloud. The left image depicts the particle distribution if only the laser range finder data is used. By taking into account the odometry when computing the proposal distribution, the particles can be drawn in a more accurate manner. As can be seen in the right image, the particle cloud is more focused, because it additionally incorporates the odometry information.

\section{The Influence of the Odometry on the Proposal}

This experiment is designed to show the advantage of the proposal distribution, which takes into account the odometry information to draw particles. In most cases, the purely laser-based proposal like the one presented in our previous approach [14] is well-suited to predict the motion of the particles. However, in a few situations the knowledge about the odometry information can be important to focus the proposal distribution. This is the case if only very poor features are available in the laser data that was used to compute the parameters of the Gaussian proposal approximation. For example, an open free space without any obstacles or a long featureless corridor can lead to high variances in the computed proposal that is only based on laser range finder data. Figure 10 illustrates this effect based on simulated laser data.

In a further experiment, we simulated a short-range laser scanner (like, e.g., the Hokuyo URG scanner). Due to the maximum range of $4 \mathrm{~m}$, the robot was unable to see the end of the corridor in most cases. This results in an high pose uncertainty in the direction of the corridor. We recorded several trajectories in this environment and used them to learn maps with and without considering the odometry when computing the proposal distribution. In this experiment, the approach considering the odometry succeeded in $100 \%$ of all cases to learn a topologically correct map. In contrast to that, our previous approach which does not take into account the odometry succeeded only in $50 \%$ of all cases. This experiment indicates the importance of the improved proposal distribution. Figure 11 depicts typical maps obtained with the different proposal distributions during this experiment. The left map contains alignment errors caused by the high pose uncertainty in the direction of the corridor. In contrast to that, a robot that also takes into account the odometry was able to maintain the correct pose hypotheses. A typical example is depicted in the right image.

Note that by increasing the number of particles, both approaches are able to map the environment correctly in $100 \%$ of all cases, but since each particle carries its own map, it is of utmost importance to keep the number of particles as low as possible. Therefore, this improved proposal is a means to limit the number of particles during mapping with RaoBlackwellized particle filters.

\section{E. Situations in Which the Scan-Matcher Fails}

As reported in Section III, it can happen that the scanmatcher is unable to find a good pose estimate based on the laser range data. In this case, we sample from the raw 

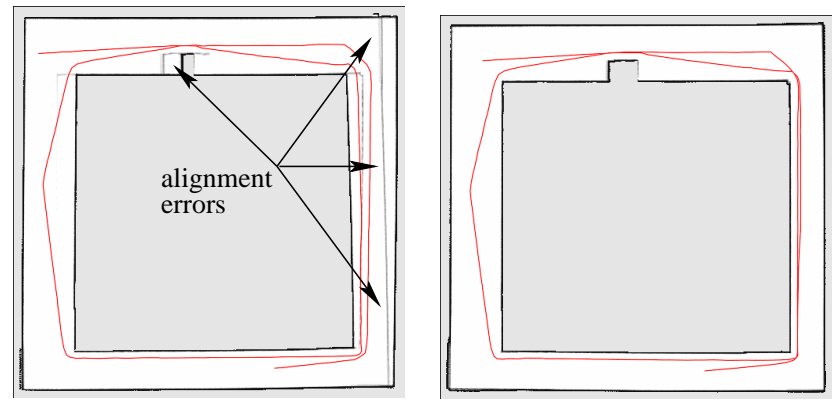

Fig. 11. Different mapping results for the same data set obtained using the proposal distribution which ignores the odometry (left image) and which considers the odometry when drawing the next generation of particles (right image).

odometry model to obtain the next generation of particles. In most tested indoor dataset, however, such a situation never occurred at all. In the MIT dataset, this effect was observed once due to a person walking directly in front of the robot while the robot was moving though a corridor that mainly consists of glass panes.

In outdoor datasets, such a situation can occur if the robot moves through large open spaces because in this case the laser range finder mainly reports maximum range readings. While mapping the Freiburg campus, the scan-matcher also reported such an error at one point. In this particular situation, the robot entered the parking area (in the upper part of the map, compare Figure 5). On that day, all cars were removed from the parking area due to construction work. As a result, no cars or other objects caused reflections of the laser beams and most parts of the scan consisted of maximum range readings. In such a situation, the odometry information provides the best pose estimate and this information is used by our mapping system to predict the motion of the vehicle.

\section{F. Runtime Analysis}

In this last experiment, we analyze the memory and computational resources needed by our mapping system. We used a standard $\mathrm{PC}$ with a $2.8 \mathrm{GHz}$ processor. We recorded the average memory usage and execution time using the default parameters that allows our algorithm to learn correct maps for nearly all real world datasets provided to us. In this setting, 30 particles are used to represent the posterior about maps and poses and a new observation, consisting of a full laser range scan, is integrated whenever the robot moved more than $0.5 \mathrm{~m}$ or rotated more than $25^{\circ}$. The Intel Research Lab dataset (see Figure 4) contains odometry and laser range readings which have been recorded over $45 \mathrm{~min}$. Our implementation required $150 M B$ of memory to store all the data using a maps with a size of approx. $40 \mathrm{~m}$ by $40 \mathrm{~m}$ and a grid resolution of $5 \mathrm{~cm}$. The overall time to correct the log file using our software was less than $30 \mathrm{~min}$. This means that the time to record a log file is around 1.5 times longer than the time to correct the log file. Table III depicts the average execution time for the individual operations.
TABLE III

AVERAGE EXECUTION TIME USING A STANDARD PC.

\begin{tabular}{|l|r|}
\hline Operation & Average Execution Time \\
\hline $\begin{array}{l}\text { Computation of the proposal distribu- } \\
\text { tion, the weights, and the map update }\end{array}$ & $1910 \mathrm{~ms}$ \\
\hline Test if resampling is required & $41 \mathrm{~ms}$ \\
\hline Resampling & $244 \mathrm{~ms}$ \\
\hline
\end{tabular}

\section{RELATED WORK}

Mapping techniques for mobile robots can be roughly classified according to the map representation and the underlying estimation technique. One popular map representation is the occupancy grid [31]. Whereas such grid-based approaches are computationally expensive and typically require a huge amount of memory, they are able to represent arbitrary objects. Feature-based representations are attractive because of their compactness. However, they rely on predefined feature extractors, which assumes that some structures in the environments are known in advance.

The estimation algorithms can be roughly classified according to their underlying basic principle. The most popular approaches are extended Kalman filters (EKFs), maximum likelihood techniques, sparse extended information filters (SEIFs), smoothing techniques, and Rao-Blackwellized particle filters. The effectiveness of the EKF approaches comes from the fact that they estimate a fully correlated posterior over landmark maps and robot poses [21, 37]. Their weakness lies in the strong assumptions that have to be made on both the robot motion model and the sensor noise. Moreover, the landmarks are assumed to be uniquely identifiable. There exist techniques [33] to deal with unknown data association in the SLAM context, however, if these assumptions are violated, the filter is likely to diverge [12]. Similar observations have been reported by Julier et al. [20] as well as by Uhlmann [43]. The unscented Kalman filter described in [20] is one way of better dealing with the non-linearities in the motion model of the vehicle.

A popular maximum likelihood algorithm computes the most likely map given the history of sensor readings by constructing a network of relations that represents the spatial constraints between the poses of the robot $[8,13,15,24]$. Gutmann et al. [15] proposed an effective way for constructing such a network and for detecting loop closures, while running an incremental maximum likelihood algorithm. When a loop closure is detected, a global optimization on the network of relation is performed. Recently, Hähnel et al. [17], proposed an approach which is able to track several map hypotheses using an association tree. However, the necessary expansions of this tree can prevent the approach from being feasible for real-time operation.

Thrun et al. [42] proposed a method to correct the poses of robots based on the inverse of the covariance matrix. The advantage of the sparse extended information filters (SEIFs) is that they make use of the approximative sparsity of the information matrix and in this way can perform predictions and updates in constant time. Eustice et al. [10] presented a technique to make use of exactly sparse information matrices in a delayed-state framework. Paskin [34] presented a solution 
to the SLAM problem using thin junction trees. In this way, he is able to reduce the complexity compared to the EKF approaches since thinned junction trees provide a linear-time filtering operation.

Folkessen et al. [11] proposed an effective approach for dealing with symmetries and invariants that can be found in landmark based representation. This is achieved by representing each feature in a low dimensional space (measurement subspace) and in the metric space. The measurement subspace captures an invariant of the landmark, while the metric space represents the dense information about the feature. A mapping between the measurement subspace and the metric space is dynamically evaluated and refined as new observations are acquired. Such a mapping can take into account spatial constraints between different features. This allows the authors to consider these relations for updating the map estimate.

Very recently, Dellaert proposed a smoothing method called square root smoothing and mapping [2]. It has several advantages compared to EKF since it better covers the nonlinearities and is faster to compute. In contrast to SEIFs, it furthermore provides an exactly sparse factorization of the information matrix.

In a work by Murphy, Doucet, and colleagues [6, 32], RaoBlackwellized particle filters (RBPF) have been introduced as an effective means to solve the SLAM problem. Each particle in a RBPF represents a possible robot trajectory and a map. The framework has been subsequently extended by Montemerlo et al. [28, 29] for approaching the SLAM problem with landmark maps. To learn accurate grid maps, RBPFs have been used by Eliazar and Parr [9] and Hähnel et al. [16]. Whereas the first work describes an efficient map representation, the second presents an improved motion model that reduces the number of required particles. Based on the approach of Hähnel et al., Howard presented an approach to learn grid maps with multiple robots [19]. The focus of this work lies in how to merge the information obtained by the individual robots and not in how to compute better proposal distributions.

Bosse et al. [1] describe a generic framework for SLAM in large-scale environments. They use a graph structure of local maps with relative coordinate frames and always represent the uncertainty with respect to a local frame. In this way, they are able to reduce the complexity of the overall problem. In this context, Modayil et al. [25] presented a technique which combines metrical SLAM with topological SLAM. The topology is utilized to solve the loop-closing problem, whereas metric information is used to build up local structures. Similar ideas have been realized by Lisien et al. [22], which introduce a hierarchical map in the context of SLAM.

The work described in this paper is an improvement of the algorithm proposed by Hähnel et al. [16]. Instead of using a fixed proposal distribution, our algorithm computes an improved proposal distribution on a per-particle basis on the fly. This allows us to directly use the information obtained from the sensors while evolving the particles. The work presented here is also an extension of our previous approach [14], which lacks the ability to incorporate the odometry information into the proposal. Especially, in critical situations in which only poor laser features for localization are available, our approach performs better than our previous one.

The computation of the proposal distribution is done in a similar way as in FastSLAM-2 presented by Montemerlo et al. [26]. In contrast to FastSLAM-2, our approach does not rely on predefined landmarks and uses raw laser range finder data to acquire accurate grid maps. Particle filters using proposal distributions that take into account the most recent observation are also called look-ahead particle filters. MoralezMenéndez et al. [30] proposed such a method to more reliably estimate the state of a dynamic system where accurate sensors are available.

The advantage of our approach is twofold. Firstly, our algorithm draws the particles in a more effective way. Secondly, the highly accurate proposal distribution allows us to utilize the effective sample size as a robust indicator to decide whether or not a resampling has to be carried out. This further reduces the risk of particle depletion.

\section{CONCLUSIONS}

In this paper, we presented an improved approach to learning grid maps with Rao-Blackwellized particle filters. Our approach computes a highly accurate proposal distribution based on the observation likelihood of the most recent sensor information, the odometry, and a scan-matching process. This allows us to draw particles in a more accurate manner which seriously reduces the number of required samples. Additionally, we apply a selective resampling strategy based on the effective sample size. This approach reduces the number of unnecessary resampling actions in the particle filter and thus substantially reduces the risk of particle depletion.

Our approach has been implemented and evaluated on data acquired with different mobile robots equipped with laser range scanners. Tests performed with our algorithm in different large-scale environments have demonstrated its robustness and the ability of generating high quality maps. In these experiments, the number of particles needed by our approach often was one order of magnitude smaller compared to previous approaches.

\section{ACKNOWLEDGMENT}

This work has partly been supported by the Marie Curie program under contract number HPMT-CT-2001-00251, by the German Research Foundation (DFG) under contract number SFB/TR-8 (A3), and by the EC under contract number FP6004250-CoSy, FP6-IST-027140-BACS, and FP6-2005-IST-5muFly. The authors would like to acknowledge Mike Bosse and John Leonard for providing us the dataset of the MIT Killian Court, Patrick Beeson for the ACES dataset, and Dirk Hähnel for the Intel Research Lab, the Belgioioso, and the Sieg-Hall dataset.

\section{REFERENCES}

[1] M. Bosse, P.M. Newman, J.J. Leonard, and S. Teller. An ALTAS framework for scalable mapping. In Proc. of the IEEE Int. Conf. on Robotics \& Automation (ICRA), pages 1899-1906, Taipei, Taiwan, 2003.

[2] F. Dellaert. Square Root SAM. In Proc. of Robotics: Science and Systems (RSS), pages 177-184, Cambridge, MA, USA, 2005. 
[3] F. Dellaert, D. Fox, W. Burgard, and S. Thrun. Monte carlo localization for mobile robots. In Proc. of the IEEE Int. Conf. on Robotics \& Automation (ICRA), Leuven, Belgium, 1998.

[4] G. Dissanayake, H. Durrant-Whyte, and T. Bailey. A computationally efficient solution to the simultaneous localisation and map building (SLAM) problem. In Proc. of the IEEE Int. Conf. on Robotics \& Automation (ICRA), pages 1009-1014, San Francisco, CA, USA, 2000.

[5] A. Doucet. On sequential simulation-based methods for bayesian filtering. Technical report, Signal Processing Group, Dept. of Engeneering, University of Cambridge, 1998.

[6] A. Doucet, J.F.G. de Freitas, K. Murphy, and S. Russel. Rao-Blackwellized partcile filtering for dynamic bayesian networks. In Proc. of the Conf. on Uncertainty in Artificial Intelligence (UAI), pages 176-183, Stanford, CA, USA, 2000.

[7] A. Doucet, N. de Freitas, and N. Gordan, editors. Sequential MonteCarlo Methods in Practice. Springer Verlag, 2001.

[8] T. Duckett, S. Marsland, and J. Shapiro. Fast, on-line learning of globally consistent maps. Journal of Autonomous Robots, 12(3):287 - 300, 2002.

[9] A. Eliazar and R. Parr. DP-SLAM: Fast, robust simultainous localization and mapping without predetermined landmarks. In Proc. of the Int. Conf. on Artificial Intelligence (IJCAI), pages 1135-1142, Acapulco, Mexico, 2003

[10] R. Eustice, H. Singh, and J.J. Leonard. Exactly sparse delayed-state filters. In Proc. of the IEEE Int. Conf. on Robotics \& Automation (ICRA), pages 2428-2435, Barcelona, Spain, 2005.

[11] J. Folkesson, P. Jensfelt, and H. Christensen. Vision SLAM in the measurement subspace. In Proc. of the IEEE Int. Conf. on Robotics \& Automation (ICRA), pages 325-330, April 2005.

[12] U. Frese and G. Hirzinger. Simultaneous localization and mapping - a discussion. In Proc. of the IJCAI Workshop on Reasoning with Uncertainty in Robotics, pages 17-26, Seattle, WA, USA, 2001.

[13] U. Frese, P. Larsson, and T. Duckett. A multilevel relaxation algorithm for simultaneous localisation and mapping. IEEE Transactions on Robotics, 21(2):1-12, 2005.

[14] G. Grisetti, C. Stachniss, and W. Burgard. Improving grid-based slam with Rao-Blackwellized particle filters by adaptive proposals and selective resampling. In Proc. of the IEEE Int. Conf. on Robotics \& Automation (ICRA), pages 2443-2448, Barcelona, Spain, 2005.

[15] J.-S. Gutmann and K. Konolige. Incremental mapping of large cyclic environments. In Proc. of the IEEE Int. Symposium on Computational Intelligence in Robotics and Automation (CIRA), pages 318-325, Monterey, CA, USA, 1999.

[16] D. Hähnel, W. Burgard, D. Fox, and S. Thrun. An efficient FastSLAM algorithm for generating maps of large-scale cyclic environments from raw laser range measurements. In Proc. of the IEEE/RSJ Int. Conf. on Intelligent Robots and Systems (IROS), pages 206-211, Las Vegas, NV, USA, 2003.

[17] D. Hähnel, W. Burgard, B. Wegbreit, and S. Thrun. Towards lazy data association in slam. In Proc. of the Int. Symposium of Robotics Research (ISRR), pages 421-431, Siena, Italy, 2003.

[18] A. Howard and N. Roy. The robotics data set repository (Radish), 2003. http://radish.sourceforge.net/.

[19] Andrew Howard. Multi-robot simultaneous localization and mapping using particle filters. In Robotics: Science and Systems, pages 201-208, Cambridge, MA, USA, 2005.

[20] S. Julier, J. Uhlmann, and H. Durrant-Whyte. A new approach for filtering nonlinear systems. In Proc. of the American Control Conference, pages 1628-1632, Seattle, WA, USA, 1995.

[21] J.J. Leonard and H.F. Durrant-Whyte. Mobile robot localization by tracking geometric beacons. IEEE Transactions on Robotics and Automation, 7(4):376-382, 1991.

[22] B. Lisien, D. Silver D. Morales, G. Kantor, I.M. Rekleitis, and H. Choset. Hierarchical simultaneous localization and mapping. In Proc. of the IEEE/RSJ Int. Conf. on Intelligent Robots and Systems (IROS), pages 448-453, Las Vegas, NV, USA, 2003.

[23] J.S. Liu. Metropolized independent sampling with comparisons to rejection sampling and importance sampling. Statist. Comput., 6:113119, 1996.

[24] F. Lu and E. Milios. Globally consistent range scan alignment for environment mapping. Journal of Autonomous Robots, 4:333-349, 1997.

[25] J. Modayil, P. Beeson, and B. Kuipers. Using the topological skeleton for scalable global metrical map-building. In Proc. of the IEEE/RSJ Int. Conf. on Intelligent Robots and Systems (IROS), pages 1530-1536, Sendai, Japan, 2004.

[26] M. Montemerlo, S. Thrun D. Koller, and B. Wegbreit. FastSLAM 2.0 An improved particle filtering algorithm for simultaneous localization and mapping that provably converges. In Proc. of the Int. Conf. on
Artificial Intelligence (IJCAI), pages 1151-1156, Acapulco, Mexico, 2003.

[27] M. Montemerlo, N. Roy, S. Thrun, D. Hähnel, C. Stachniss, and J. Glover. CARMEN - the carnegie mellon robot navigation toolkit. http://carmen.sourceforge.net, 2002.

[28] M. Montemerlo and S. Thrun. Simultaneous localization and mapping with unknown data association using FastSLAM. In Proc. of the IEEE Int. Conf. on Robotics \& Automation (ICRA), pages 1985-1991, Taipei, Taiwan, 2003.

[29] M. Montemerlo, S. Thrun, D. Koller, and B. Wegbreit. FastSLAM: A factored solution to simultaneous localization and mapping. In Proc. of the National Conference on Artificial Intelligence (AAAI), pages 593598, Edmonton, Canada, 2002

[30] R. Morales-Menéndez, N. de Freitas, and D. Poole. Real-time monitoring of complex industrial processes with particle filters. In Proc. of the Conf. on Neural Information Processing Systems (NIPS), pages 14331440, Vancover, Canada, 2002.

[31] H.P. Moravec. Sensor fusion in certainty grids for mobile robots. AI Magazine, pages 61-74, Summer 1988.

[32] K. Murphy. Bayesian map learning in dynamic environments. In Proc. of the Conf. on Neural Information Processing Systems (NIPS), pages 1015-1021, Denver, CO, USA, 1999.

[33] J. Neira and J.D. Tardós. Data association in stochastic mapping using the joint compatibility test. IEEE Transactions on Robotics and Automation, 17(6):890-897, 2001

[34] M.A. Paskin. Thin junction tree filters for simultaneous localization and mapping. In Proc. of the Int. Conf. on Artificial Intelligence (IJCAI), pages 1157-1164, Acapulco, Mexico, 2003.

[35] M.K. Pitt and N. Shephard. Filtering via simulation: auxilary particle filters. Technical report, Department of Mathematics, Imperial College, London, 1997.

[36] N. Roy, M. Montemerlo, and S. Thrun. Perspectives on standardization in mobile robot programming. In Proc. of the IEEE/RSJ Int. Conf. on Intelligent Robots and Systems (IROS), pages 2436-2441, Las Vegas, NV, USA, 2003

[37] R. Smith, M. Self, and P. Cheeseman. Estimating uncertain spatial realtionships in robotics. In I. Cox and G. Wilfong, editors, Autonomous Robot Vehicles, pages 167-193. Springer Verlag, 1990.

[38] C. Stachniss and G. Grisetti. Mapping results obtained with Rao-Blackwellized particle filters. http://www.informatik.unifreiburg.de/ stachnis/research/rbpfmapper/, 2004.

[39] S. Thrun. An online mapping algorithm for teams of mobile robots. Int. Journal of Robotics Research, 20(5):335-363, 2001.

[40] S. Thrun, W. Burgard, and D. Fox. Probabilistic Robotics, chapter Robot Perception, pages 171-172. MIT Press, 2005.

[41] S. Thrun, W. Burgard, and D. Fox. Probabilistic Robotics, chapter Robot Motion, pages 121-123. MIT Press, 2005.

[42] S. Thrun, Y. Liu, D. Koller, A.Y. Ng, Z. Ghahramani, and H. DurrantWhyte. Simultaneous localization and mapping with sparse extended information filters. Int. Journal of Robotics Research, 23(7/8):693-716, 2004.

[43] J. Uhlmann. Dynamic Map Building and Localization: New Theoretical Foundations. $\mathrm{PhD}$ thesis, University of Oxford, 1995.

[44] R. van der Merwe, N. de Freitas, A. Doucet, and E. Wan. The unscented particle filter. Technical Report CUED/F-INFENG/TR380, Cambridge University Engineering Department, August 2000. 